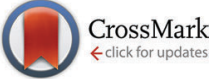

Cite this: Phys. Chem. Chem. Phys., 2014, 16, 21988

Received 1st August 2014, Accepted 1st September 2014

DOI: $10.1039 / c 4 c p 03439 b$

www.rsc.org/pccp

\title{
Short versus long chain polyelectrolyte multilayers: a direct comparison of self-assembly and structural properties $\dagger$
}

\author{
Samantha Micciulla, ${ }^{a}$ Samuel Dodoo, $\ddagger^{a}$ Chloé Chevigny, $\S^{a}$ André Laschewsky ${ }^{b c}$ and \\ Regine von Klitzing ${ }^{\star a}$
}

\begin{abstract}
Successful layer-by-layer (LbL) growth of short chain ( 30 repeat units per chain) poly(sodium styrene sulfonate) (PSS)-poly(diallyl dimethylammonium chloride) (PDADMAC) multilayers is presented for the first time and compared with the growth of equivalent long chain polyelectrolyte multilayers (PEMs). A detailed study performed by quartz crystal microbalance with dissipation (QCM-D) is carried out and three main processes are identified: (i) initial mass uptake, (ii) adsorption-desorption during layer equilibration and (iii) desorption during rinsing. In contrast to the high stability and strong layer increment of high molecular weight (HMW) PEMs, layer degradation characterizes low molecular weight (LMW) multilayers. In particular, two different instability phenomena are observed: a constant decrease of sensed mass during equilibration after PDADMAC adsorption, and a strong mass loss by salt-free rinsing after PSS adsorption. Yet, an increase of salt concentration leads to much stronger layer growth. First, when the rinsing medium is changed from pure water to $0.1 \mathrm{M} \mathrm{NaCl}$, the mass loss during rinsing is reduced, irrespective of molecular weight. Second, an increase in salt concentration in the LMW PE solutions causes a larger increment during the initial adsorption step, with no effect on the rinsing. Finally, the mechanical properties of the two systems are extracted from the measured frequency and dissipation shifts, as they offer a deeper insight into the multilayer structures depending on chain length and outermost layer. The paper enriches the field of PE assembly by presenting the use of very short $\mathrm{PE}$ chains to form multilayers and elucidates the role of preparation conditions to overcome the limitation of layer stability.
\end{abstract}

\section{Introduction}

Since the introduction of self-assembled polyelectrolyte multilayers (PEMs) in the earlier nineties, ${ }^{1}$ intense studies have been carried out to understand the fundamental rules governing their buildup and properties. ${ }^{2-5}$ Ease of preparation and extreme versatility make them great candidates for the design of functional and responsive coatings for biomedical technologies, ${ }^{6}$

\footnotetext{
${ }^{a}$ Stranski-Laboratorium, Institut für Chemie, Technische Universität Berlin, Strasse des 17. Juni 124,D-10623 Berlin, Germany.E-mail: klitzing@chem.tu-berlin.de

${ }^{b}$ Institut für Chemie, Universität Potsdam, Karl-Liebknechtstr. 24-25, 14476 Postdam-Golm, Germany

${ }^{c}$ Fraunhofer-Institut für Angewandte Polymerforschung, Geiselbergstraße 69, 14476 Potsdam-Golm, Germany

$\dagger$ Electronic supplementary information (ESI) available. See DOI: 10.1039/ c4cp03439b

\$ Current address: Kwame Nkrumah University of Science and Technology, Accra Rd, Kumasi, Ghana.

$\S$ Current address: INRA, UR1268 Biopolymeres, Interactions et Assemblages, F-44316 Nantes, France.
}

microcapsules for drug delivery, ${ }^{7}$ substrates for cell adhesion and proliferation, ${ }^{8}$ solid-state light-emitting devices. ${ }^{9}$ However, the simplicity of PEMs assembly contrasts with the complex interplay of parameters which determines film growth and structure. In addition to the nature of polyelectrolyte pair used, charge density, rinsing medium, preparation method (dipping, spin-coating, spraying), temperature, $\mathrm{pH}$ and ionic strength strongly affect the final system properties. ${ }^{10-17}$

The investigation of such effects relies on the use of very sensitive techniques, being able to detect in situ changes of the order of nanometers or nanograms. For this purpose, in the last decade the use of quartz crystal microbalance with dissipation (QCM-D) has been established for the study of multilayer assembly, structure and responsive properties. ${ }^{18-21}$ Detailed reviews offer an exhaustive overview on the topic. ${ }^{22-24}$ The advantage of QCM-D is that both adsorbed amount and layer viscoelasticity can be monitored. The former is obtained from the shift of oscillation frequency $(\Delta f)$ of the substrate-coated quartz crystal, the latter from the energy dissipated by the system $(\Delta D)$ during the shear mode oscillation. The combination of 
both measured shifts offers an insight into assembly and structural properties of the adsorbed film.

Despite the large amount of literature available on PEMs, the role of chain length has been rarely investigated, ${ }^{25-33}$ in particular neglecting the oligomeric range. The few studies demonstrate that short chain polymers ${ }^{28,34}$ or small molecules ${ }^{35}$ tend to form rather unstable systems lacking monotonous film growth, opposite to the behavior generally observed for long chain polymers. In particular, Sui et al. ${ }^{36}$ studied the assembly of poly(styrene sulfonate) (PSS)-poly(4-vinylmethylpyridine) (P4VMP) multilayers from high molecular weight (HMW) and low molecular weight (LMW) polymers. They noticed a reduced thickness increment when HMW chains $\left(10^{5} \mathrm{~g}^{-10 l^{-1}}\right)$ were combined with LMW ones $\left(10^{4} \mathrm{~g} \mathrm{~mol}^{-1}\right)$, and no PEM growth for pure short chain PEs at any of the investigated salt concentrations (from 0 to $1 \mathrm{M}$ ). Similar findings were reported for the layer-by-layer (LbL) assembly of poly(aminoacid)s. ${ }^{28}$ These results were explained by the formation of soluble polyelectrolyte complexes (PECs) at the solid/liquid interface, leading to stripping from the multilayer surface. For short chain PEs this process is faster than the formation of more polymer-polymer contacts, which instead promote the formation of a stable polymer blend. Moreover, chain entanglements are prevented by the low molar mass, therefore surface polyelectrolytes are easily stripped off to form bulk complexes. ${ }^{36}$ The formation of water soluble polyelectrolyte complexes from PEMs causing layer degradation was reviewed by Sukhishvili et $a .^{37}$ and discussed by several authors. In fact, the equilibration of PECs from the multilayer to the bulk in condition of low charge density $^{38}$ or excess of charges ${ }^{39}$ may lead to post-adsorption film rupture. As mentioned by Haynie et al., ${ }^{28}$ both kinetics and thermodynamics favor the formation of soluble complexes, since the gain in entropy of released counterions does not compensate the loss of translational entropy by adsorbing in the multilayer phase. Therefore, the exchange of complexes between film and solution may occur within experimental times. As a result of that, stable multilayering of pairs of oligomeric electrolytes has been reported so far only in exceptional cases, typically requiring additional interactions, such as hydrophobic or $\pi$ - $\pi$-stacking forces ${ }^{40-45}$ in addition to electrostatic ones.

Recently, we reported on the first characterization of a 4-layer poly(sodium styrene sulfonate)-poly(diallyl dimethylammonium chloride) (PSS-PDADMAC) PEM made of very short chain (LMW, $\sim 30$ repeat units per chain) polyelectrolytes and the comparison with numerical simulations. ${ }^{46}$ Therein it was pointed out that the equilibration process following the initial mass adsorption is the weak step for layer stability. In fact, chain detachment takes place within experimental times, limiting the layer growth.

Our interest towards the oligomeric PEMs is motivated by the possibility of studying processes which are kinetically hindered for long chain systems due to layer interdigitation, and of creating a new class of materials with different chain dynamics and layer structure. Therefore we extended the investigation of LMW PEs assembly to 12-layer-systems, with a detailed analysis in situ of the adsorption process by QCM-D.
The results clarify the crucial aspects of PEM assembly which determine the stability of adsorbed layers over a full adsorptionrinse cycle. Being aware of the strong influence of the preparation conditions on multilayer structure, the effect of salt concentration of rinsing medium and PE solution on growth and stability of LMW PEMs was investigated. The direct comparison with the classical high molecular weight (HMW, $\sim 300$ repeat units per chain) PSS-PDADMAC system highlights structural differences responsible for the distinct layer increment and stability. The role of chain dynamics is discussed as the key to enhance the layer-bylayer growth of short chain multilayers.

\section{Materials and methods}

\subsection{Materials}

HMW and LMW linear poly(diallyl dimethyl ammonium chloride) (PDADMAC, $72000 \mathrm{~g} \mathrm{~mol}^{-1}$, PDI = 1.75 and $5000 \mathrm{~g} \mathrm{~mol}^{-1}$, PDI = 1.5) were synthesized by free radical polymerization, as described in a previous work. ${ }^{47}$ HMW poly(styrene sulfonate sodium salt) (PSS, $70000 \mathrm{~g} \mathrm{~mol}^{-1}$, PDI = 2.5) was purchased from Sigma Aldrich (Germany) and LMW PSS $6520 \mathrm{~g} \mathrm{~mol}^{-1}$, PDI < 1.2) from Polymer Standard Service, Germany. Poly(ethylene imine) (PEI, $750000 \mathrm{~g} \mathrm{~mol}^{-1}$ ) was from Sigma-Aldrich (Germany). $\mathrm{NaCl}$ (purity $>99.9 \%$ ) from Merck (Germany) was used to vary the ionic strength of polyelectrolyte solutions. All the reagents were used as received without any further purification.

\subsection{Layer-by-layer assembly}

Polyelectrolyte solutions $0.01 \mathrm{~mol}(\mathrm{mon}) \mathrm{L}^{-1}$ were prepared in $0.1 \mathrm{M} \mathrm{NaCl}$ solution for both MWs and also in $0.5 \mathrm{M} \mathrm{NaCl}$ for LMW ones. Prior to multilayer deposition, gold-coated quartz crystals (QSX301, Q-Sense, Sweden) were cleaned by a mixture of $\mathrm{NH}_{3}: \mathrm{H}_{2} \mathrm{O}_{2}: \mathrm{H}_{2} \mathrm{O}(1: 1: 5, \mathrm{v} / \mathrm{v})$ for $10 \mathrm{~min}$ at $75{ }^{\circ} \mathrm{C}$, then rinsed with large excess of Milli-Q water $\left(18 \mathrm{M} \Omega \mathrm{cm}^{-1}\right)$ and blown dry by $\mathrm{N}_{2}$ stream. A precursor PEI layer was adsorbed on the gold substrate before multilayer assembly, followed by alternate PSSPDADMAC adsorption-rinse cycles. The adsorption time was set to $5 \mathrm{~min}$ to limit the layer degradation observed during LMW PDADMAC adsorption (Fig. S1, ESI $\dagger$ ), followed by 5 min rinsing with either salt-free water or $\mathrm{NaCl}$ solution. For the sake of comparison, long chain multilayers were prepared using the same protocol. All the preparations were carried out at $(25 \pm 0.02){ }^{\circ} \mathrm{C}$ with solution flow rate of $0.1 \mathrm{~mL} \mathrm{~min}^{-1}$.

\subsection{Quartz crystal microbalance with dissipation}

QCM-D monitors the change of resonance frequency of a substrate-coated quartz crystal upon mass adsorption. In brief, a quartz crystal sandwiched between two gold electrodes is brought to vibrate at the resonance frequency by applying a proper alternating voltage across its thickness. From the amplitude of the freely decaying damped oscillation

$$
A(t)=A_{0} \sin (2 \pi f t+\psi) \exp (-t / \tau)
$$

the resonance frequency $f$ and decay time constant $\tau$ are obtained, ${ }^{48}$ with $A_{0}$ the amplitude of oscillation at $t=0$ and $\psi$ 
the phase shift. The dissipation factor $D$ is obtained from the relation $D=1 / \pi f \tau$.

Under the assumption that a rigid film is adsorbed on the resonator, the shift of resonance frequency $\Delta f$ and the sensed mass $\Delta m$ are linearly dependent, as stated by the Sauerbrey relation ${ }^{49}$

$$
\Delta f=-\frac{2 f_{0}^{2}}{A \sqrt{\rho_{\mathrm{q}} \mu_{\mathrm{q}}}} \Delta m
$$

where $f_{0}$ is the fundamental frequency of quartz $(4.95 \mathrm{MHz}$ for AT cut quartz sensors), $A$ the piezoelectrically active area, $\rho_{\mathrm{q}}$ the quartz density $\left(2.648 \mathrm{~g} \mathrm{~cm}^{-3}\right)$ and $\mu_{\mathrm{q}}$ the quartz shear modulus $\left(2.947 \times 10^{-11} \mathrm{~g} \mathrm{~cm}^{-1} \mathrm{~s}^{-2}\right)$. Measurements were run on the instrument E1 from Q-Sense (Sweden), the software Q-Soft from Q-Sense was used for data acquisition.

Viscoelastic modeling of $\Delta f$ and $\Delta D$ on a Voigt element allows the determination of shear modulus, viscosity and hydrodynamic thickness of film from specific relations, as reported by Voinova et al.: ${ }^{50}$

$$
\begin{gathered}
\Delta f \sim-\frac{1}{2 \pi \rho_{\mathrm{q}} h_{\mathrm{q}}}\left\{\frac{\eta_{1}}{\delta_{\mathrm{l}}}+h_{\mathrm{f}} \rho_{\mathrm{f}} \omega-2 h_{\mathrm{f}}\left(\frac{\eta_{1}}{\delta_{\mathrm{l}}}\right)^{2} \frac{\eta_{\mathrm{f}} \omega^{2}}{\mu_{\mathrm{f}}^{2}+\omega^{2} \eta_{\mathrm{f}}^{2}}\right\} \\
\Delta D \sim+\frac{1}{2 \pi f \rho_{\mathrm{q}} h_{\mathrm{q}}}\left\{\frac{\eta_{1}}{\delta_{\mathrm{l}}}+2 h_{\mathrm{f}}\left(\frac{\eta_{1}}{\delta_{\mathrm{l}}}\right)^{2} \frac{\mu_{\mathrm{f}} \omega}{\mu_{\mathrm{f}}^{2}+\omega^{2} \eta_{\mathrm{f}}^{2}}\right\}
\end{gathered}
$$

with $h_{\mathrm{q}}$ thickness of the crystal, $\eta_{1}$ and $\delta_{1}$ viscosity and viscous penetration depth of the shear wave in the bulk medium, $\rho_{\mathrm{f}}$ film density, $h_{\mathrm{f}}$ film thickness, $\mu_{\mathrm{f}}$ film shear modulus and $\eta_{\mathrm{f}}$ film viscosity. The data elaboration was performed using the software Q-Tools provided by the instrument supplier. The multilayers were modeled as a laterally homogeneous film in a purely Newtonian bulk liquid. The film density was fixed to 1000 and $1020 \mathrm{~kg} \mathrm{~m}^{-3}$ for HMW and LMW multilayers, respectively, fluid density $997 \mathrm{~kg} \mathrm{~m}^{-3}$ and fluid viscosity to $0.9 \mathrm{MPa} \mathrm{s} .^{20,21}$ Loss $\left(G^{\prime \prime}\right)$ and storage $\left(G^{\prime}\right)$ moduli were calculated according to the relation ${ }^{51}$

$$
G^{*}=\mu_{\mathrm{f}}+i 2 \pi f \eta_{\mathrm{f}}=G^{\prime}+i G^{\prime \prime}
$$

\section{Results}

In the first part of this section, the multilayer assembly of HMW and LMW polyelectrolytes from $0.1 \mathrm{M} \mathrm{NaCl}$ solution and salt-free rinsing is presented, with focus on the change of mass during layer equilibration and rinse step. In the second part, the preparation of PEMs from the same PE solution but rinsing with $0.1 \mathrm{M} \mathrm{NaCl}$ is reported. The last part focuses on the effect of salt concentration in the PE solution for the adsorption of short chains from solutions prepared at the same monomer concentration in $0.5 \mathrm{M} \mathrm{NaCl}$.

\subsection{Effect of chain length on PEMs assembly and structure}

A typical example of frequency shift $(\Delta f)$ and dissipation change $(\Delta D)$ for the adsorption-salt-free rinse cycle of PSS-PDADMAC layers is shown in Fig. 1, where 5th (PSS) and 6th (PDADMAC) layers are reported. These may represent the behavior of PSS-PDADMAC bilayers when the substrate effect vanishes and polyion-polyion interactions become more relevant. The complete preparation can be found in Fig. S2 of the ESI. $\dagger$ From the reported results and according to literature, ${ }^{52}$ three kinetic processes control the layer growth: (i) an initial strong mass adsorption, (ii) the chain rearrangements during layer equilibration, to achieve a more stable conformation, and (iii) the mass loss during rinsing. In case of LMW PEMs, chain reorganization leads to desorption and layer erosion after PDADMAC adsorption. This indicates that the formation of soluble PECs is favored compared to lateral or vertical reorganization typical for HMW PEMs. ${ }^{52}$

Further mass removal occurs during rinsing with salt-free water. Normally the lost material consists of loosely bound polymers and counterions, but the strong removal during rinsing after LMW PSS deposition suggests a deeper layer instability.

The changes of $\Delta f$ from adsorption to rinsing cycle are coupled to changes of energy dissipation: $\Delta D$ decreases to
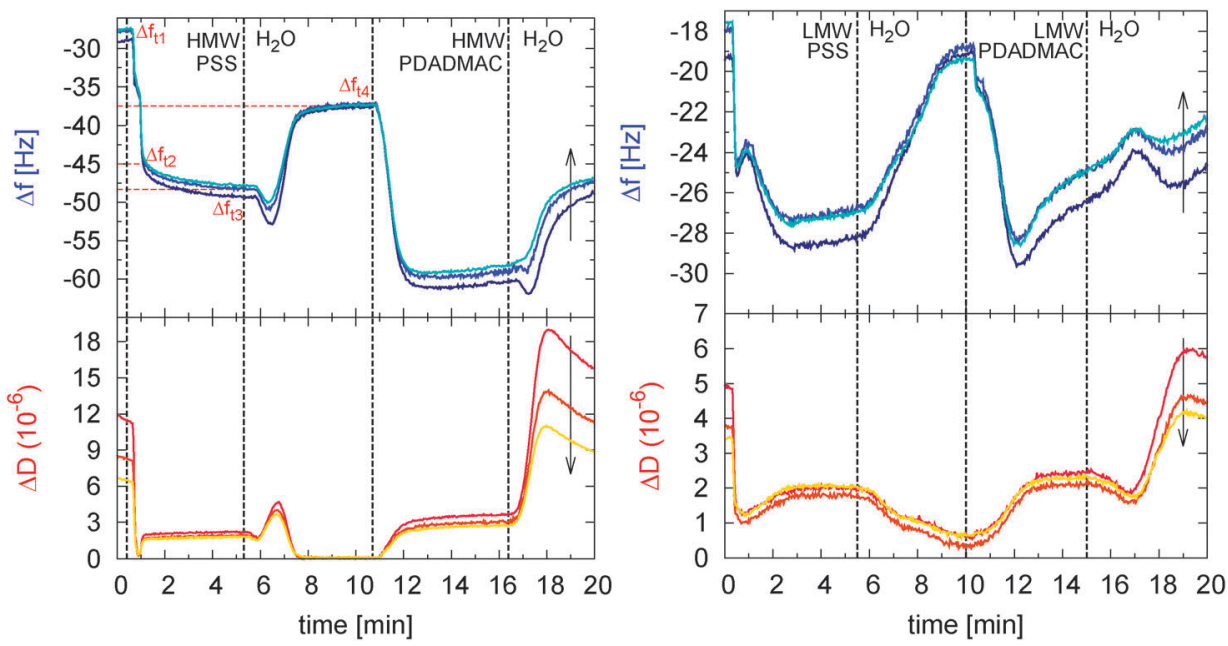

Fig. 1 Frequency shift and dissipation recorded during the adsorption of HMW and LMW PSS-PDADMAC layers. Multilayers were prepared from PE solutions $0.01 \mathrm{~mol}(\mathrm{mon}) \mathrm{L}^{-1}$ in $0.1 \mathrm{M} \mathrm{NaCl}$, rinsing with salt-free water. Arrows point to increasing overtone number (5th, 7th and 9 th). Due to sake of clarity, two layers (5th and 6th) are reported. The full curve is shown in Fig. S2 of the ESI. $\dagger$ 
lower values by rinsing after PSS deposition for both MWs, but increases by a factor of 3 up to 10 after PDADMAC adsorption (Fig. 1). These changes are attributed to layer shrinking and layer swelling, respectively. ${ }^{20}$ In fact, the decrease of dissipation after PSS deposition indicates the formation of a poorly hydrated and rather stiff layer, implying a highly intrinsically compensated PEM. In contrast, the dissipation increase after PDADMAC adsorption indicates a softer and well hydrated layer. Such an alternation of swollen and collapsed states agrees with findings reported in literature ${ }^{21,31}$ and is independent of the chain length.

Besides the hint for the viscoelastic character of the adsorbed layer, the splitting of different overtones points out some features of the multilayer structure. As higher overtones have shorter penetration depth, they are more sensitive to regions closer to the substrate, while lower overtone numbers propagate deeper away from the substrate. ${ }^{53}$ Therefore the trend $\Delta D_{5}>$ $\Delta D_{7}>\Delta D_{9}$, where $\Delta D_{n}$ is the dissipation shift for the $n$th overtone, means that a higher amount of water is coupled at the polymer/liquid interface, causing higher dissipative losses, whereas a more compressed structure is present closer to the substrate. Similar results are reported in previous works on HMW PEMs ${ }^{21,54}$ and also by Schönhoff et al. in ref. 55 and references therein.

Layer viscosity and shear modulus reveal the mechanical properties of a film. The validity of a viscoelastic modeling to describe a system from QCM-D measurements is closely related to the ratio between adsorbed mass and dissipation. A general approximation states that when the value of $\Delta D / \Delta f$ is $\ll 0.4 \times$ $10^{-6} \mathrm{~s}$, the response of the adsorbed mass is mainly elastic. ${ }^{23}$ This approach was used here to verify the validity of viscoelastic modeling (Fig. S5 in the ESI $\dagger$ ). Layer viscosity $\eta_{\mathrm{f}}$ and layer shear modulus $\mu_{\mathrm{f}}$ were obtained from the fit of $\Delta f$ and $\Delta D$ on a Voigt element through the modeling software provided by the QCM-D supplier. The parameters were fixed as described in the experimental section. Storage $\left(G^{\prime}\right)$ and loss $\left(G^{\prime \prime}\right)$ moduli were calculated according to eqn (5), and they were used to verify whether the system reacts with elastic $\left(G^{\prime \prime} / G^{\prime}<1\right)$, viscous $\left(G^{\prime \prime} / G^{\prime}>1\right)$ or viscoelastic $\left(G^{\prime \prime} / G^{\prime} \approx 1\right)$ behavior $^{23}$ to the shear deformation. Aware of the more elastic character of PSS-terminated PEMs, as the values of $\Delta D / \Delta f$ during water rinsing after PSS adsorption reaches a very low values, we found a rather good agreement between experimental and fitting data. The obtained viscoelastic parameters after salt-free rinsing are reported in Fig. 2 and support a pure qualitative discussion on buildup and structure of PSS-PDADMAC layers.

Both $\eta_{\mathrm{f}}$ and $\mu_{\mathrm{f}}$ show an odd-even effect with increasing number of adsorption steps irrespective of the chain length. The higher viscosity of PSS-terminated PEMs indicates the presence of dense, polymer-rich layers in contrast to loose and more hydrated ones when terminated by PDADMAC. The same oscillations are observed for the shear modulus, which is correlated to the density of complexation sites among polyions ${ }^{51}$ and therefore determines the elastic behavior of a polymer network. The higher shear modulus $\mu_{\mathrm{f}}$ of PSS layers suggests a higher density of chain entanglements compared to PDADMAC-terminated PEMs,

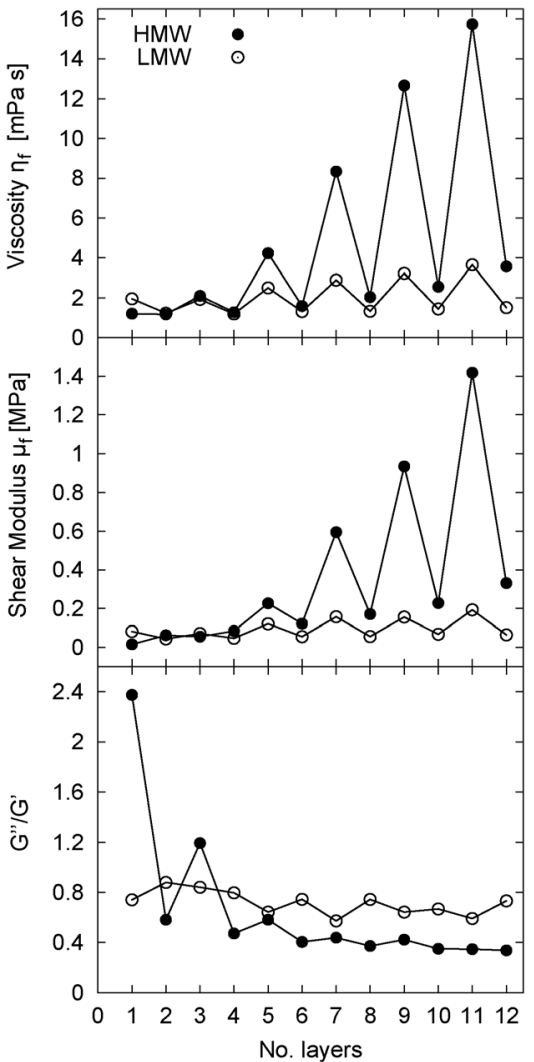

Fig. 2 Viscosity, shear modulus and viscoelastic ratio $G^{\prime \prime} / G^{\prime}$ for HMW and LMW PEMs as a function of layer number. Odd layer numbers correspond to PSS, even ones to PDADMAC. The "zero" is the positive PEl precursor layer. The data represent the layer properties after salt-free rinsing.

which give higher elastic modulus. Although the QCM-D data can not unequivocally determine the PEM structure, the proposed models are supported by analogous findings from determination of the Young modulus of PDADMAC-PSS PEMs by nanoindentation. ${ }^{56}$

Another difference between long and short chain multilayers is the increase of viscosity and shear modulus with layer number for HMW versus the almost constant values for LMW ones. This may be explained by a structural change, namely an enhanced network density, of long chain PEMs through subsequent layer deposition, and the stratification of thin layers with constant mechanical properties for short chain ones. However, for both chain lengths the elastic behavior dominates the system response to the applied shear stress, as demonstrated by $G^{\prime \prime} / G^{\prime}<1$.

\subsection{Effect of salt concentration of rinsing medium on PEM assembly}

To investigate the effect of rinsing solution on layer structure and stability, long and short chain multilayers were prepared from the same PE solutions as reported in the previous section, but the rinsing step was performed with $0.1 \mathrm{M} \mathrm{NaCl}$ instead of with pure water.

Fig. 3 shows $\Delta f$ and $\Delta D$ of 5 th (PSS) and 6th (PDADMAC) layers as representative examples of bilayer growth. The complete 

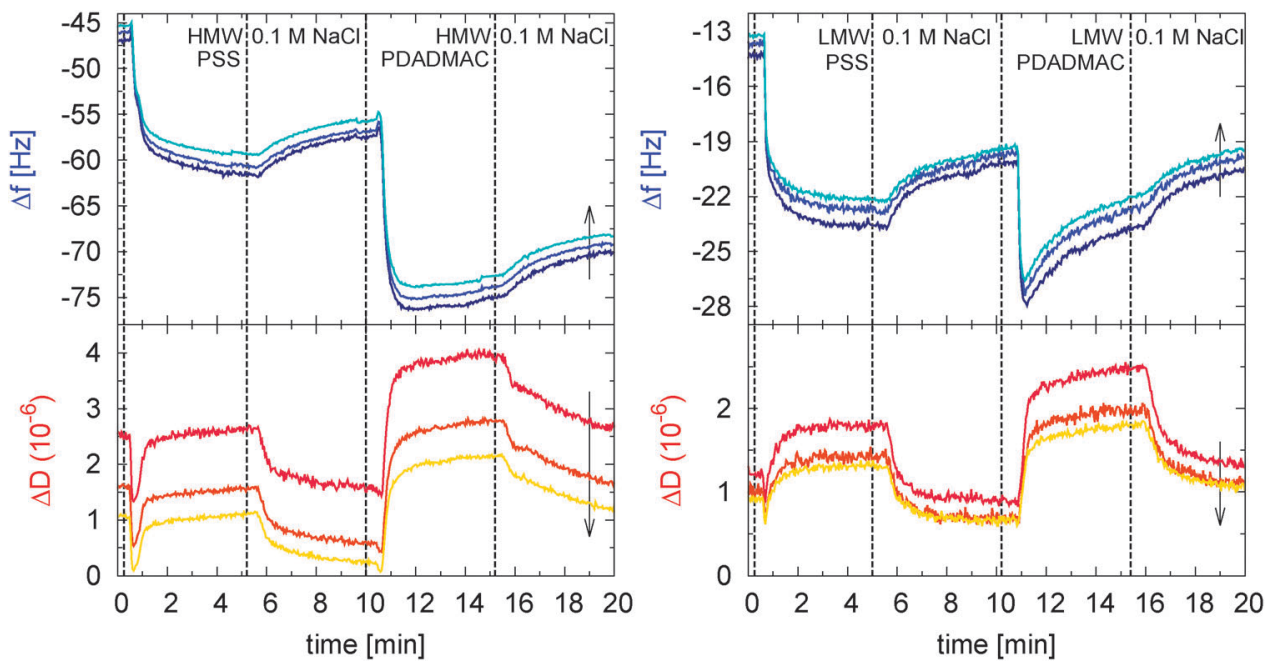

Fig. 3 Frequency shift and dissipation for the adsorption of HMW and LMW PSS-PDADMAC layers. Multilayers were prepared from PE solutions $0.01 \mathrm{~mol}(\mathrm{mon}) \mathrm{L}^{-1}$ in $0.1 \mathrm{M} \mathrm{NaCl}$, rinsing with $0.1 \mathrm{M} \mathrm{NaCl}$. Arrows point to increasing overtone number (5th, 7th and 9th). Due to sake of clarity, two layers (5th and 6th) are reported. The full curve is shown in Fig. S3 of the ESI. $\dagger$

multilayer preparation is presented in Fig. S3 of the ESI. $\dagger$ The change of rinsing medium does not help to limit the layer degradation during the adsorption of LMW PDADMAC, which remains very pronounced. However, the mass removal during rinsing is reduced. Although the lower loss has to account for a reduced water removal from the layer, most of the contribution can be attributed to the polymer. In fact, a previous experiment showed that by changing the solution in contact with the PEM from pure water to $\mathrm{NaCl} 0.1 \mathrm{M}$ and vice versa, the measured frequency shifts were limited between 1 and $2 \mathrm{~Hz}$.

Another interesting element to mention is the splitting of dissipation for different overtones. This may due to a structural inhomogeneity between inner and outer parts of the multilayer. As a matter of fact, the higher dissipation recorded for lower overtones corresponds to ion-rich and hydrated outermost layers, in contrast to a more compact structure of the inner part, as previously reported by reflectivity and diffusion studies. ${ }^{57,58}$

A viscoelastic modeling was not applied in this case due to the very low $\Delta D / \Delta f$ ratio for all layers (Fig. S5 in the ESI $\dagger$ ).

\subsection{Effect of salt concentration of PE solution on PEM assembly}

The effect of ionic strength of polyelectrolyte solution on assembly and stability of LMW PEMs was studied by monitoring their LbL growth from solutions with $0.5 \mathrm{M} \mathrm{NaCl}$ and saltfree rinsing.

Fig. 4 shows $\Delta f$ and $\Delta D$ for the adsorption-rinse cycle of 5 th (PSS) and 6th (PDADMAC) short chain layers; the full multilayer preparation is reported in Fig. S4 of the ESI. $\dagger$

As demonstrated by the more negative frequency shift and in agreement with previous findings on HMW PEMs,${ }^{57,59,60}$ higher mass adsorption is achieved when PEs are deposited from solutions at higher ionic strength. The stronger mass uptake

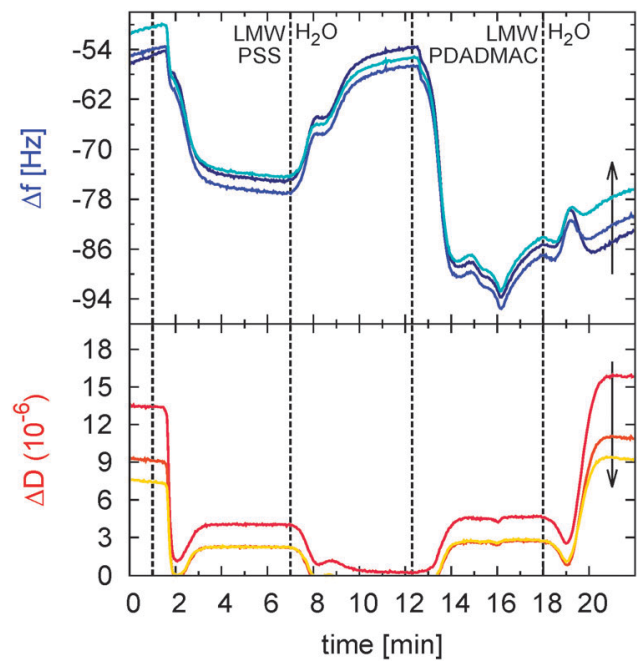

Fig. 4 Frequency shift and dissipation registered during the adsorption of HMW and LMW PSS-PDADMAC layers. Multilayers were prepared from PE solutions $0.01 \mathrm{~mol}(\mathrm{mon}) \mathrm{L}^{-1}$ in $0.5 \mathrm{M} \mathrm{NaCl}$, rinsing with salt-free water. Arrows point to increasing overtone number (5th, 7th and 9th). Due to sake of clarity, two layers (5th and 6th) are reported. The full curve is shown in Fig. S4 of the ESI. $\dagger$

is explained by the reduced chain repulsion due to higher extrinsic charge compensation, and to the larger surface available due to the adsorption of more coiled chains. The layer erosion which starts after the adsorption of LMW PDADMAC is in this case a smaller part of the initially adsorbed mass if compared to the degradation from $0.1 \mathrm{M} \mathrm{NaCl}$, reported in Fig. 1. Such a reduced erosion of the preformed layer is a crucial aspect, which improves the growth of short chain multilayers. The weak element of this preparation protocol remains the strong mass removal by rinsing after PSS deposition, which is still very high and limits its layer increment over the full adsorption-rinse cycle. 


\section{Discussion}

\subsection{Effect of salt concentration during layer rinsing}

The challenge of growing multilayers from very short polyelectrolyte chains is related to the competition of opposite phenomena: on one hand, the adsorption at the interface and diffusion into the PEM; on the other hand, the layer erosion which limits the system growth. To focus on these processes, the relative change of sensed mass $\Delta(\Delta m)$ during layer equilibration was calculated by

$$
\Delta(\Delta m)[\%]=\frac{\Delta f_{t 3}-\Delta f_{t 2}}{\Delta f_{t 2}-\Delta f_{t 1}} \times 100
$$

where $\Delta f_{t n}$ corresponds to the frequency shift at the specific experimental times, namely at $t 1$, when PE solution is injected, at $t 2$, when the initial fast adsorption is completed, and at $t 3$, when the equilibration process is interrupted. An example of the mentioned frequency shifts $\Delta f_{t n}$ is shown in Fig. 1 . The same was done for the rinsing process,

$$
\Delta(\Delta m)[\%]=\frac{\Delta f_{t 4}-\Delta f_{t 3}}{\Delta f_{t 2}-\Delta f_{t 1}} \times 100
$$

where $t 4$ is the time when the rinsing process is completed. Eqn (6) and (7) are based on the assumption of a rigid polymer layer coupled to the resonator, for which holds $\Delta m \propto \Delta f$. This approximation is supported by the low $\Delta D / \Delta f$ ratio (Fig. S5 in ESI $\dagger$ ).

Fig. 5 shows the relative mass change $\Delta(\Delta m)$ during layer equilibration and rinsing for HMW and LMW PEMs prepared at low ionic strength $(0.1 \mathrm{M})$ and by rinsing either with salt-free water or by $0.1 \mathrm{M} \mathrm{NaCl}$. The advantage of comparing the relative change of sensed mass is that this accounts for the different amount of water carried by the two chain lengths. From Fig. 5a it can be seen that there is always a gain of mass during equilibration after HMW PSS adsorption, irrespective of the rinsing medium. Based on the diffusion model reported for PEMs ${ }^{61,62}$ and on the distribution of extrinsic charge compensation described by Schlenoff and coworkers for PDADMAC-PSS multilayers, ${ }^{63}$ this secondary mass adsorption can be explained by the presence of a reservoir of mobile PDADMAC chains in the multilayer, which diffuse back to the interface during PSS deposition and enhance the uptake. In the case of HMW PDADMAC, a small loss is measured during layer equilibration, which could be due to some water release upon complexation between the polyions. ${ }^{31}$ The different chain dynamics of the two polymers may be reasoned by their intra-charge distance, around $0.25 \mathrm{~nm}$ and $0.6 \mathrm{~nm}$ for PSS $^{64}$ and PDADMAC, ${ }^{65}$ respectively. This causes a more flat conformation for PSS upon adsorption, and the formation of bigger loops and extrinsically compensated structure for PDADMAC. ${ }^{63,66}$ The higher extrinsic charge compensation of the polycation allows the diffusion towards the inner part of the multilayer, due to a weaker interaction with oppositely charged polyion. Accordingly, an excess of positive charges inside the PEM after PDADMAC deposition has been reported, in contrast to the strong intrinsic charge compensation after PSS adsorption. ${ }^{63}$

In the case of LMW PEs, neither PSS nor PDADMAC shows a secondary uptake during layer equilibration. On the contrary, a loss of mass is observed, in particular during the equilibration which follows PDADMAC adsorption. This process can be explained as the result of two effects. First of all, the number of charged groups per chain is not high enough to give a stable adsorption on PEM surface. It is likely that the low gain in entropy upon counterion release is not sufficient to compensate for the entropic loss from bulk to solid phase, therefore chain detachment is promoted. Moreover, as addressed previously in literature, ${ }^{10,14,67}$ a minimum charge density is needed to achieve stable layer adsorption. Although both long and short chain PEs presented in this work have 100\% charge density, the adsorption of very short chains may lead to poor charge overcompensation, which weakens the interaction with the next adsorbed layer. Secondly, the formation of charged PECs by the stripping of previously adsorbed chains has to be considered.

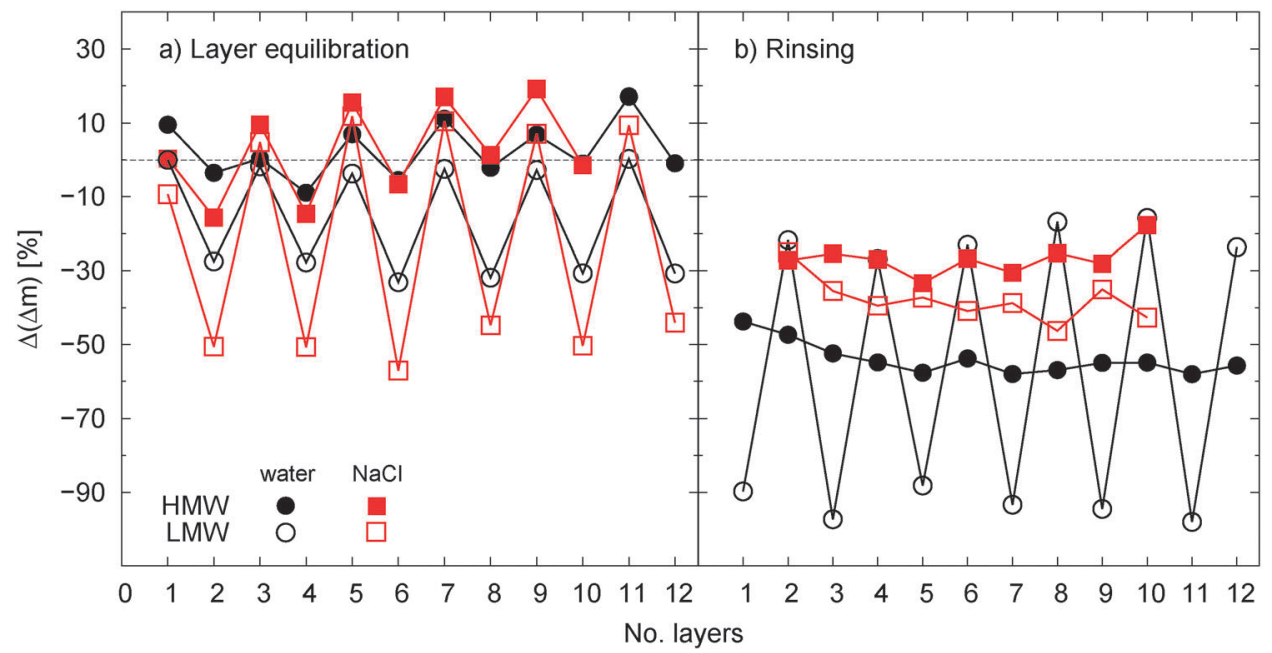

Fig. 5 Relative mass change during (a) layer equilibration (eqn (6)) and (b) rinsing (eqn (7)) for HMW and LMW multilayers prepared with salt-free water and $0.1 \mathrm{M} \mathrm{NaCl}$ rinsing solutions. Data points at odd layer numbers correspond to PSS termination, even ones to PDADMAC termination. 
The asymmetric charge distance of the two polyelectrolytes and the excess of charge from counterions enhance the feasibility of this process, as pointed out in previous investigations on the formation of soluble interpolyelectrolyte complexes. ${ }^{68}$ Furthermore, the formation of very thin layers due to the short chain length and the poor PDADMAC adsorption may hinder the internal diffusion. As a consequence, the absence of a polycation reservoir prevents the secondary uptake for LMW PSS during equilibration.

From the mentioned aspects on the adsorption mechanism of HMW and LMW PEs and according to previous findings, it can be stated that (i) for polymers with high linear charge density and small intrinsic persistence length, the adsorption at the surface is favored by a strong intrinsic charge compensation; (ii) for polymers with lower linear charge density and larger intrinsic persistence length, either internal diffusion or chain detachment is promoted.

The use of $\mathrm{NaCl}$ rinsing instead of pure water between subsequent PE adsorption steps influences the chain dynamics independently of the molecular weight. For HMW PEMs, a small increment of PSS uptake and a reduction of PDADMAC loss during equilibration is obtained (Fig. 5), and both effects contribute to a higher layer growth. In contrast, the $\mathrm{NaCl}$ rinsing has opposite effects on LMW PEs: on one hand, it induces an additional mass uptake after the initial PSS adsorption; on the other hand, it causes a stronger loss during equilibration after PDADMAC adsorption. To explain both the secondary PSS uptake and the enhanced PDADMAC instability, the effect of the rinsing medium on the internal composition of PEM should be considered. It is likely that by rinsing with salt solution instead of with pure water, a higher counterion content is kept in the outermost layer. This weakens the interactions between oppositely charged polyions, and therefore enhances the internal diffusion of adsorbing chains. At the same time, the weaker polyion-polyion interaction enhances the detachment of LMW PDADMAC or the formation of soluble PECs, which results in a stronger degradation after the initial PDADMAC adsorption. This proves that the influence of the rinsing medium is not limited to the rinse step, because it determines structure and ionic content of the adsorbed layer and therefore the efficiency of the next adsorption step.

By analyzing the relative change of mass during rinsing with respect to the initially adsorbed amount (Fig. 5b), a reduction from $50 \%$ to $30 \%$ for HMW PEs is observed when the PEM is washed with $\mathrm{NaCl}$ solution instead of with pure water. In the case of LMW PEs, the change of rinsing medium from salt-free water to $\mathrm{NaCl}$ solution has a crucial effect on layer stability, as it allows to reduce the mass loss after PSS adsorption from $90 \%$ to $40 \%$ of the initial uptake. Although the lower removal needs to account for a reduced water loss, it is very likely that a higher amount of chains are kept inside the layer, since the suppression of an abrupt change of osmotic pressure at the film/liquid interface would reduce the layer destabilization. A much smaller loss is measured during rinsing after PDADMAC deposition (around 20\% of the initially adsorbed mass). Yet considering the amount removed during layer equilibration, the total mass loss is around $50 \%$, i.e. comparable to the amount washed from HMW.

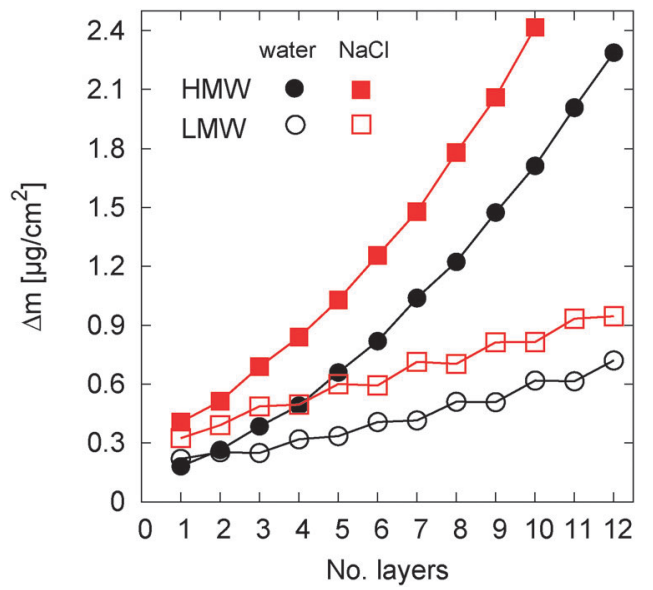

Fig. 6 Sensed mass for increasing number of deposited layers. Films were prepared from PE solutions $0.01 \mathrm{~mol}(\mathrm{mon}) \mathrm{L}^{-1}$ in $0.1 \mathrm{M} \mathrm{NaCl}$. Reported data refer to the multilayer thickness after rinsing with salt-free water or $0.1 \mathrm{M} \mathrm{NaCl}$. Odd layer numbers represent PSS termination, even ones PDADMAC termination. The "zero" corresponds to the positive PEI precursor layer.

From the results discussed above it appears clear that layer destabilization is the limiting factor for the growth of LMW PEMs. The degradation of PDADMAC layer occurs already during layer equilibration, while in the case of LMW PSS the salt-free rinsing triggers a strong mass removal. The latter can be reduced by $\mathrm{NaCl}$ rinsing, since besides the reduced counterions and water removal, the absence of an abrupt change of osmotic pressure at the PEM interface may avoid the stripping of loosely bound chains or complexes.

As the LbL growth of a multilayer is the result of adsorption, equilibration and rinsing step, the sensed mass $\Delta m$ per layer measured over a complete preparation cycle is the most representative parameter to describe the system's growth. Fig. 6 shows $\Delta m$ as a function of layer number for both MWs and rinsing protocols.

The mass increment is governed by a non-linear increase for HMW multilayers and a linear increase for LMW ones. For the latter, the linear regime may result from the stratification of very thin layers, having a lower water content, and from the strong degradation, which keeps the adsorbed amount very low.

Despite this difference between the two systems, the assembly by $\mathrm{NaCl}$ rinsing gives higher layer increment for both chain lengths. In particular for LMW PEMs this is proved by the slope of a linear fit on the experimental data (Fig. S6 in the ESI $\dagger$ ), which is higher for preparation by $\mathrm{NaCl}$ rinsing.

As a result of the balance between layer destabilization and growth, in the case of LMW PEMs the rinsing protocol has a different effect on the mass increment of each polyelectrolyte: when the short chain PEMs are prepared with pure water, the strong mass removal during rinsing after PSS adsorption causes a higher increment for PDADMAC over a full adsorption-rinse cycle. On the contrary, the preparation with $\mathrm{NaCl}$ rinsing induces a stronger destabilization of PDADMAC layers but enhanced stability of PSS ones, which results in a higher increment for PSS layers. 


\subsection{Effect of salt concentration during layer assembly}

The influence of ionic strength in the PE solution was also analyzed for the growth of LMW PEMs. The relative change of sensed mass $\Delta(\Delta m)$ is reported in Fig. 7 for layer equilibration and salt-free rinsing. The data for the preparation at low ionic strength were presented in Fig. 5 and are reported here for the sake of comparison.

Fig. 7a shows that beyond the 4th layer the PSS uptake is enhanced and the PDADMAC loss is reduced during layer equilibration. Analogous to PEM preparation with $\mathrm{NaCl}$ rinse, the secondary PSS adsorption is explained by the diffusion of PDADMAC chains first into the multilayer and then back to the interface, which enhances PSS adsorption. This process is more likely at higher ionic strength due to the higher counterion condensation around the chains, which weaken the interaction among polyions. However, in condition of excess of charges the formation of PECs should be also favored. ${ }^{37}$ To verify that, the absolute change of mass during equilibration was considered and compared to the adsorption at low ionic strength (Fig. S7 of ESI $\dagger$ ). As a matter of fact, the mass loss during PDADMAC equilibration is enhanced at higher ionic strength, when the extrinsic charge compensation is also higher. The measured signal will be also including the hydration water carried by the stripped chains or complexes, which may be higher in this condition than at low ionic strength. However, Ellipsometry measurements performed in ambient condition on the same kind of systems assembled on silicon wafer proves the increase of the adsorbed amount at higher salt concentration, leading to thicker LMW PEMs (Fig. S8 in ESI $\dagger$ ). The importance of this result is to demonstrate that the reduced degradation of PDADMAC layers is due to the enhanced initial PE adsorption.

As demonstrated by Fig. 7b, the rinsing step has no influence on the layer stability of the LMW PEMs prepared at different ionic strength. In fact, in both cases the most prominent effect is the strong destabilization of PSS layer when the PE solution is changed with pure water for rinsing. Nevertheless, in contrast to

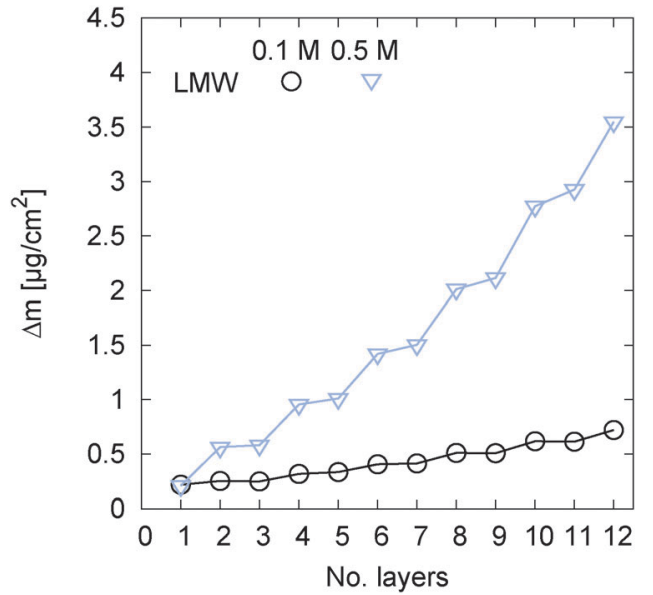

Fig. 8 Sensed mass as a function of number of layers. LMW PEMs were prepared from PE solutions $0.01 \mathrm{~mol}(\mathrm{mon}) \mathrm{L}^{-1}$ in $0.1 \mathrm{M}$ or $0.5 \mathrm{M} \mathrm{NaCl}$. Reported data refer to the sensed mass after rinsing with salt-free water. Odd numbers represent PSS termination, even ones PDADMAC termination. The "zero" corresponds to the positive PEI precursor layer.

assembly at low ionic strength, such a strong loss is balanced by the enhanced PSS uptake during equilibration, and the higher initial PDADMAC adsorption. These two factors have a strong impact on the layer increment, as proved by the stronger PEM growth shown in Fig. 8.

All the processes discussed above demonstrate the complex interplay of effects dominating assembly and stability of polyelectrolyte multilayers. The proper choice of preparation conditions is crucial to limit layer instability and achieve an efficient LbL growth. In Fig. 9 a schematic summary of the effect of salt concentration of rinsing medium and PE solution on the change of mass during equilibration and rinsing for both long and short chain (PSS-PDADMAC) multilayers is offered. It may serve as a general guide for the choice of assembly protocol to enhance the layer growth.

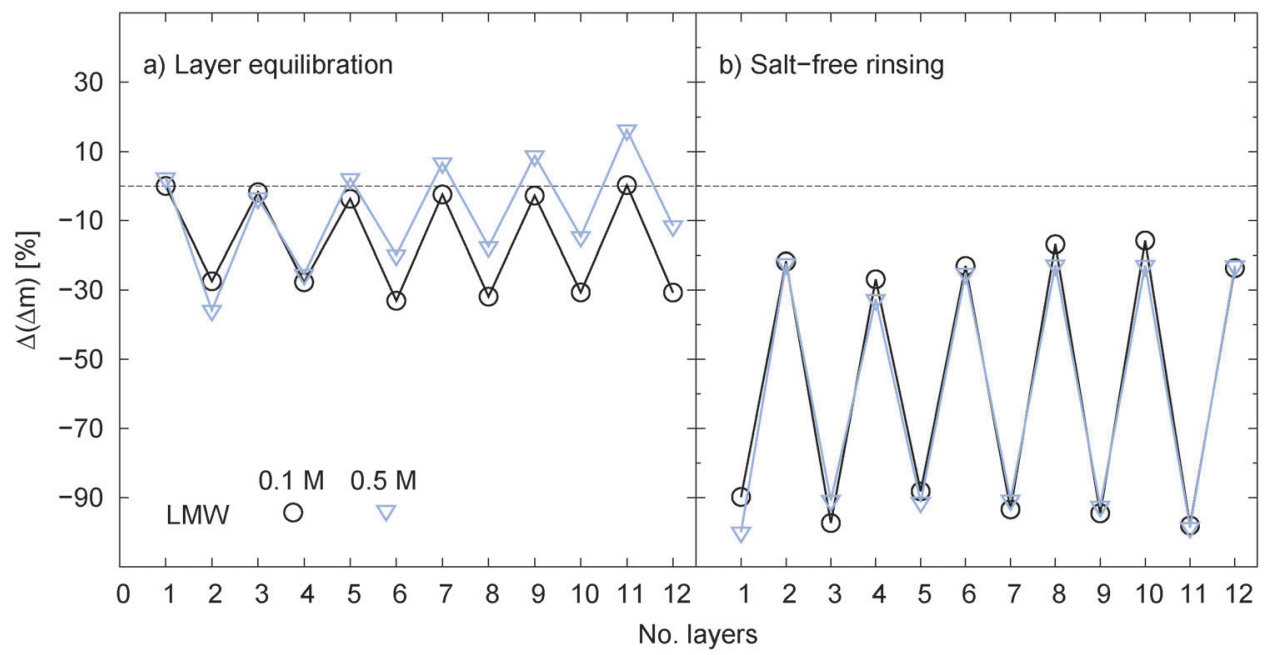

Fig. 7 Relative mass change during (a) layer equilibration and (b) rinsing for HMW and LMW multilayers prepared at 0.1 and $0.5 \mathrm{M} \mathrm{NaCl}$. Rinsing was performed by salt-free water. Odd numbers refer to PSS termination, even ones to PDADMAC termination. 


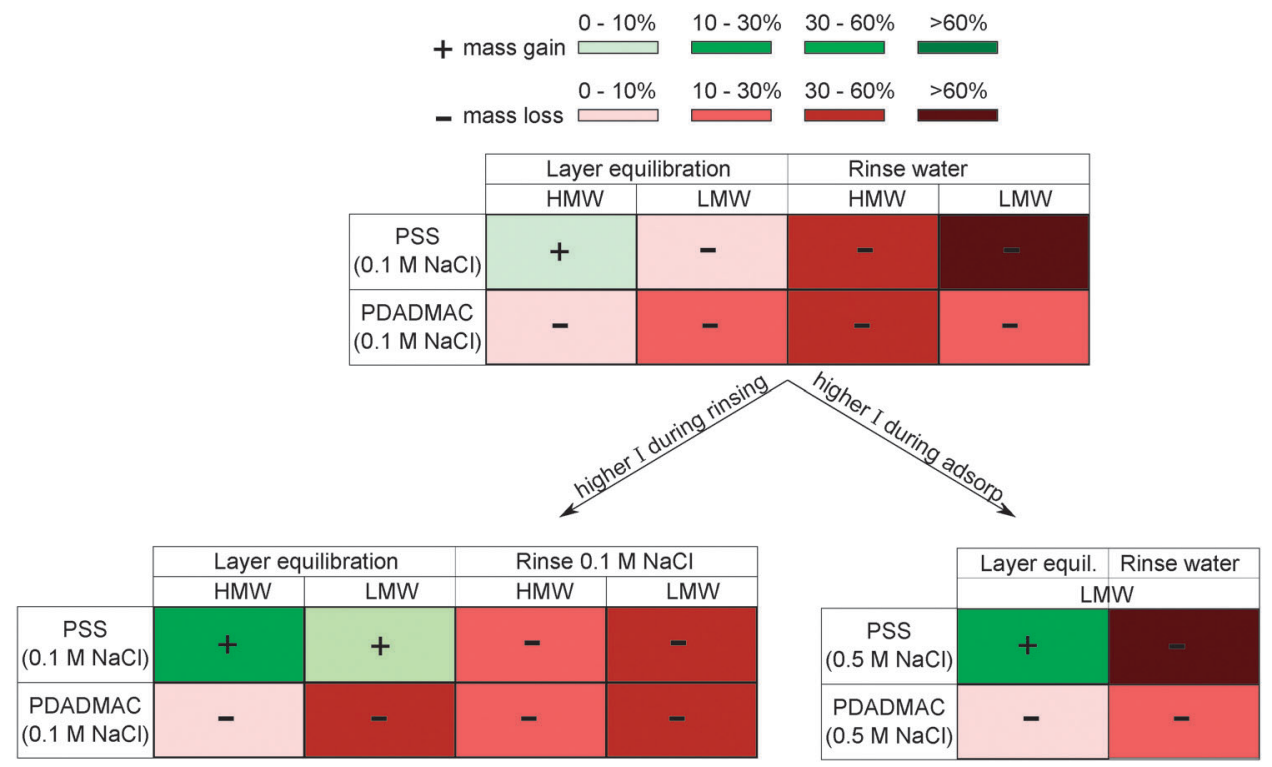

Fig. 9 Schematic summary of the effect of increasing salt concentration either in the rinsing solution (bottom, left), or in the PE solution (bottom, right) on the relative change of mass during layer equilibration and rinsing for HMW and LMW PEMs. The symbols + and - indicate gain and loss of mass, respectively. The different color intensity from light to dark corresponds to increasing percentage of mass change. The reported value account for both polymer and coupled water. The reported scheme may serve as a guide for the choice of assembly conditions to achieve higher layer growth.

\subsection{Mechanism of multilayering at low ionic strength: HMW versus LMW PEs assembly}

The change of shear modulus $\mu_{\mathrm{f}}$ and viscosity $\eta_{\mathrm{f}}$ during multilayer assembly reveals some fundamental properties of the adsorbed layers, namely the formation of highly intrinsic compensated PSS layers (high elastic modulus and high viscosity) in contrast to the swollen and more extrinsic compensated PDADMAC layers (low shear modulus and low viscosity). These properties are valid for both HMW and LMW multilayers, which means that scaling down to shorter chain length does not change the rules governing buildup and behavior of PSS-PDADMAC systems.

However, assumed that the viscoelastic modeling of such films suffers of strong approximations like constant layer density or the stronger elastic contribution, it is possible to deduce some general difference between the two systems. In particular, in the case of short chain PEMs the mechanical properties are mostly constant, which suggests the stratification of oppositely charged layers with a constant inner structure. In contrast, an increase of $\mu_{\mathrm{f}}$ and $\eta_{\mathrm{f}}$ with layer number for the long chain system indicates an enhancement of layer density and entanglements. The proposed structures for HMW and LMW PEMs are schematically represented in Fig. 10, consisting of (i) an entangled polymer network and (ii) a layered alternation of oppositely charged layers, respectively. The structural model of short chain PEMs agree with the one proposed by Nestler et $a .^{32}$ for the assembly of low molecular weight PEs.

\subsection{The role of PEC structure on the stability of LMW PEM}

The assembly of short chain multilayers was previously reported by Schlenoff and coworkers $^{36}$ for the pair poly(methyl vinyl pyridine) (PM4VP)-poly(styrene sulfonate) (PSS) ( $\sim 40$ repeat units per chain). The multilayering was investigated over a wide

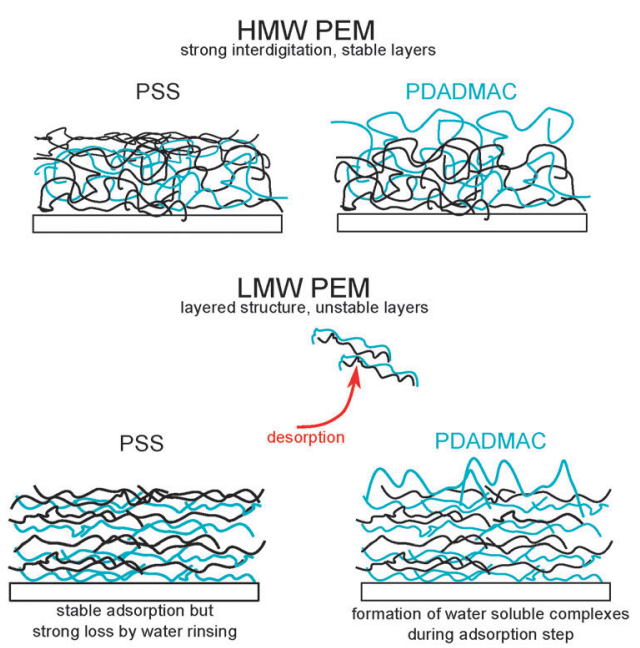

Fig. 10 Schematic summary of the main structural features of HMW and LMW chain PEMs. A highly interdigitated system is deduced for long chain PEMs and a layered alternation of oppositely charged layers for short chain ones. Stiff and intrinsically compensated layers are formed after PSS adsorption as the layer collapses during water rinsing. On the contrary, layer swelling during salt-free rinsing and the formation of extrinsically compensated layers is obtained for PDADMAC. In contrast to the stable adsorption of HMW PEs, LMW ones have to account for polymer desorption. This occurs by the strong mass loss during water rinsing after PSS adsorption and the formation of soluble PE complexes during PDADMAC adsorption. The length of the drawn segments does not correlate to the true chain lengths

range of salt concentration, but no relevant multilayer growth was observed. Considering the aforementioned work as a comparison to the present study, there are two important differences that are worth to be mentioned: 
(1) Polyelectrolyte pair. The polyelectrolyte pair is a crucial parameter determining the growth efficiency. As the polyions PSS and P4VMP have very similar structure and intra-chain charge distance, their combination leads to the formation of highly intrinsically compensated pairs, therefore very thin and flat layers. The resulting complexes are also more hydrophobic (i.e. lower hydration, lower extrinsic charge compensation) than PDADMAC-PSS ones. ${ }^{69}$ As the results reported in the previous section demonstrate that chain diffusion is a key mechanism to enhance the polymer uptake and counteract the layer degradation, the higher persistence length of PDADMAC compared to PSS and the rather high hydrophilicity of PSS-PDADMAC complexes lead to the formation of rougher layers compared to PSS-P4VMP, allowing for a higher chain mobility through the structure.

(2) Drying between adsorption steps. Irreversible structural changes may be induced by the drying process after layer deposition. This potentially reduces layer porosity and smooth the outermost layer, preventing diffusive processes through the multilayer. This idea is supported by the findings of Tedeschi et $a l^{35}$ on the adsorption of dyes inside a multilayer. They found that the uptake of a dye was reduced when the adsorption steps were alternated by drying with argon. This result was explained in terms of structural changes which reduce layer porosity and therefore the diffusion of the dye to the inner part of the PEM. In our work, no drying steps were performed between subsequent depositions, therefore the chain diffusion inside the multilayers was not hindered.

\section{Conclusions}

In contrast to previous findings and to common expectations, low molecular weight polyelectrolytes can be successfully assembled by layer-by-layer routes to prepare multilayers. Herein it is shown that the formation of unstable layers occurs due to the low number of complexation sites per chain and by the formation of soluble PECs, which diffuse back to the bulk phase. The first multilayer degradation process takes place during layer equilibration after LMW PDADMAC adsorption. Differently, LMW PSS layers are more stable during the equilibration step, but subject to strong mass loss during rinsing with salt-free water. Therefore for short chains PEs, at least one of the two processes, either the reorganization during equilibration or the rinsing, leads to strong losses of mass and prevents a straight layer increment during multilayer built-up.

A variety of effects on the chain dynamics are observed when increasing the salt content either in the rinsing medium, or in the PE solution. In the first case, the rinse cycle with salt solution instead of with pure water decreases the mass loss after PSS adsorption. In addition, the diffusion of PDADMAC chains into the multilayer is enhanced, due to the higher extrinsic charge compensation. This leads to the formation of a bigger reservoir of polycations, which increases the PSS uptake during the next adsorption cycle. In the second case, the higher salt concentration in the PE solution enhances the initial mass uptake during adsorption for both PEs. Although the mass loss during layer equilibration and salt-free rinsing is not reduced, the higher amounts of adsorbed PE balances the layer erosion and leads to higher layer increments.
The possibility of preparing multilayers with very short PE chains has huge implications for the fundamental research and for the applications of these systems. Besides a number of analogous properties to HMW PEMs, the faster dynamics of LMW PEMs allows to follow processes, which are normally hidden by the kinetic irreversibility of long chain PEs assembly, on experimental time scale.

Further on, the possibility of extending the investigation of multilayers to the oligomeric level may help to validate theoretical simulations on PE assembly, ${ }^{46,70}$ for which the computational load of large macromolecules is still out of reach, or to generalize experimental results ${ }^{71}$ to a broader range of molecular weights. In terms of applications, using different chain lengths might be a tool to control the stability of PEM membranes, e.g. for uptake and release of drugs.

\section{Acknowledgements}

The Deutsche Forschungsgemeinschaft DPG is acknowledged for the financial support within the Priority Program SPP 1369.

\section{References}

1 G. Decher, J. D. Hong and J. Schmitt, Thin Solid Films, 1992, 210-211, 831-835.

2 S. T. Dubas and J. B. Schlenoff, Macromolecules, 1999, 32, 8153-8160.

3 P. Bertrand, A. Jonas, A. Laschewsky and R. Legras, Macromol. Rapid Commun., 2000, 21, 319-348.

4 E. Blomberg, E. Poptoshev and F. Caruso, Langmuir, 2006, 22, 4153-4157.

5 J. B. Schlenoff and S. T. Dubas, Macromolecules, 2001, 34, 592-598.

6 R. R. Costa and J. a. F. Mano, Chem. Soc. Rev., 2014, 43, 3453-3479.

7 W. C. Mak, K. Y. Cheung and D. Trau, Chem. Mater., 2008, 20, 5475-5484.

8 O. V. Semenov, A. Malek, A. G. Bittermann, J. Vörös and A. H. Zisch, Tissue Eng., Part A, 2009, 15, 2977-2990.

9 A. Wu, D. Yoo, J. Lee and M. Rubner, J. Am. Chem. Soc., 1999, 121, 4883-4891.

10 R. Steitz, W. Jaeger and R. von Klitzing, Langmuir, 2001, 17, 4471-4474.

11 J. E. Wong, H. Zastrow, W. Jaeger and R. von Klitzing, Langmuir, 2009, 25, 14061-14070.

12 U. Voigt, W. Jaeger, G. H. Findenegg and R. von Klitzing, J. Phys. Chem. B, 2003, 107, 5273-5280.

13 R. von Klitzing, J. E. Wong, W. Jaeger and R. Steitz, Curr. Opin. Colloid Interface Sci., 2004, 9, 158-162.

14 K. Glinel, A. Moussa, A. M. Jonas and A. Laschewsky, Langmuir, 2002, 18, 1408-1412.

15 R. Köhler, I. Dönch, P. Ott, A. Laschewsky, A. Fery and R. Krastev, Langmuir, 2009, 25, 11576-11585.

16 P. Ott, J. Gensel, S. Roesler, K. Trenkenschuh, D. Andreeva, A. Laschewsky and A. Fery, Chem. Mater., 2010, 22, 3323-3331. 
17 M. Koetse, A. Laschewsky, A. M. Jonas and W. Wagenknecht, Langmuir, 2002, 18, 1655-1660.

18 A. Garg, J. R. Heflin, H. W. Gibson and R. M. Davis, Langmuir, 2008, 24, 10887-10894.

19 N. M. Alves, C. Picart and J. F. Mano, Macromol. Biosci., 2009, 9, 776-785.

20 Z. Feldötö, I. Varga and E. Blomberg, Langmuir, 2010, 26, 17048-17057.

21 J. J. Iturri Ramos, S. Stahl, R. P. Richter and S. E. Moya, Macromolecules, 2010, 43, 9063-9070.

22 D. Johannsmann, Phys. Chem. Chem. Phys., 2008, 10, 4516.

23 I. Reviakine, D. Johannsmann and R. P. Richter, Anal. Chem., 2011, 83, 8838-8848.

24 M. C. Dixon, J. Biomol. Tech., 2008, 19, 151-158.

25 M. Lösche, J. Schmitt, G. Decher, W. G. Bouwman, K. Kjaer and M. Lo, Macromolecules, 1998, 31, 8893-8906.

26 S. Schwarz, J. Nagel and W. Jaeger, Macromol. Symp., 2004, 211, 201-216.

27 M. Houska, E. Brynda and K. Bohatá, J. Colloid Interface Sci., 2004, 273, 140-147.

28 D. T. Haynie, S. Balkundi, N. Palath, K. Chakravarthula and K. Dave, Langmuir, 2004, 20, 4540-4547.

29 H. Zhang, D. Wang, Z. Wang and X. Zhang, Eur. Polym. J., 2007, 43, 2784-2791.

30 B. Sun, C. M. Jewell, N. J. Fredin and D. M. Lynn, Langmuir, 2007, 23, 8452-8459.

31 X. Liu, K. K. Goli, J. Genzer and O. J. Rojas, Langmuir, 2011, 27, 4541-4550.

32 P. Nestler, M. Passvogel and C. A. Helm, Macromolecules, 2013, 46, 8-15.

33 K. Buecher, K. Graf, H. Ahrens and C. A. Helm, Langmuir, 2002, 18, 3585-3591.

34 L. Kolarik, D. Furlong, H. Joy, C. Struijk and R. Rowe, Langmuir, 1999, 15, 8265-8275.

35 C. Tedeschi and F. Caruso, J. Am. Chem. Soc., 2000, 122, 5841-5848.

36 Z. Sui, D. Salloum and J. B. Schlenoff, Langmuir, 2003, 19, 2491-2495.

37 S. A. Sukhishvili, E. Kharlampieva and V. Izumrudov, Macromolecules, 2006, 39, 8873-8881.

38 D. Kovacevic and S. V. D. Burgh, Langmuir, 2002, 7058-7063.

39 H. Mjahed, J.-C. Voegel, A. Chassepot, B. Senger, P. Schaaf, F. Boulmedais and V. Ball, J. Colloid Interface Sci., 2010, 346, 163-171.

40 A. Hatzor, T. Moav, H. Cohen, S. Matlis, J. Libman, A. Vaskevich, A. Shanzer and I. Rubinstein, J. Am. Chem. Soc., 1998, 120, 13469-13477.

41 T. Tang, A. Herrmann, K. Peneva, K. Müllen and S. E. Webber, Langmuir, 2007, 23, 4623-4628.

42 J. Locklin, K. Shinbo, K. Onishi, F. Kaneko, Z. Bao and R. C. Advincula, Chem. Mater., 2003, 15, 1404-1412.

43 M. Pyrasch and B. Tieke, Langmuir, 2001, 17, 7706-7709.

44 D. H. Kim, J. L. Hernandez-Lopez, J. Liu, G. Mihov, L. Zhi, R. E. Bauer, D. Grebel-Köhler, M. Klapper, T. Weil, K. Müllen, S. Mittler and W. Knoll, Macromol. Chem. Phys., 2005, 206, 52-58.
45 M. Salomäki, M. Räsänen, J. Leiro, T. Huti, M. Tenho, J. Lukkari and J. Kankare, Adv. Funct. Mater., 2010, 20, 2140-2147.

46 S. Micciulla, P. A. Sánchez, J. Smiatek, B. Qiao, M. Sega, A. Laschewsky, C. Holm and R. von Klitzing, Soft Mater., 2014, DOI: 10.1080/1539445x.2014.930046.

47 H. Dautzenberg, E. Gornitz and W. Jaeger, Macromol. Chem. Phys., 1998, 199, 1561-1571.

48 M. Rodahl and B. Kasemo, Rev. Sci. Instrum., 1996, 67, 3238.

49 G. Sauerbrey, Z. Phys., 1959, 155, 206-222.

50 M. V. Voinova, M. Rodahl, M. Jonson and B. Kasemo, Phys. Scr., 1999, 59, 391-396.

51 M. Lundin, F. Solaqa, E. Thormann, L. Macakova and E. Blomberg, Langmuir, 2011, 27, 7537-7548.

52 E. Guzmán, H. A. Ritacco, F. Ortega and R. G. Rubio, J. Phys. Chem. C, 2012, 116, 15474-15483.

53 N.-J. Cho, K. K. Kanazawa, J. S. Glenn and C. W. Frank, Anal. Chem., 2007, 79, 7027-7035.

54 O. M. Tanchak, K. G. Yager, H. Fritzsche, T. Harroun, J. Katsaras and C. J. Barrett, Langmuir, 2006, 22, 5137-5143.

55 M. Schönhoff, V. Ball, A. R. Bausch, C. Dejugnat, N. Delorme, K. Glinel, R. von Klitzing and R. Steitz, Colloids Surf., A, 2007, 303, 14-29.

56 A. Lehaf, H. Hariri and J. Schlenoff, Langmuir, 2012, 28, 6348-6355.

57 R. Steitz, V. Leiner, R. Siebrecht and R. von Klitzing, Colloids Surf., A, 2000, 163, 63-70.

58 P. Nazaran, V. Bosio, W. Jaeger, D. F. Anghel and R. v. Klitzing, J. Phys. Chem. B, 2007, 111, 8572-8581.

59 X. Gong and C. Gao, Phys. Chem. Chem. Phys., 2009, 11, 11577-11586.

60 Y. Lvov, K. Ariga, M. Onda, I. Ichinose and T. Kunitake, Colloids Surf., A, 1999, 146, 337-346.

61 P. Lavalle, C. Picart, J. Mutterer, C. Gergely, H. Reiss, J.-C. Voegel, B. Senger and P. Schaaf, J. Phys. Chem. B, 2004, 108, 635-648.

62 C. Porcel, P. Lavalle, G. Decher, B. Senger, J.-C. Voegel and P. Schaaf, Langmuir, 2007, 23, 1898-1904.

63 R. A. Ghostine, M. Z. Markarian and J. B. Schlenoff, J. Am. Chem. Soc., 2013, 135, 7636-7646.

64 R. von Klitzing, R. Köhler and C. Chevigny, Neutron Reflectometry at Polyelectrolyte Multilayers, Wiley-VCH Verlag GmbH \& Co. KGaA, 2012, pp. 219-268.

65 K. W. Mattison, P. L. Dubin and I. J. Brittain, J. Phys. Chem. $B, \mathbf{1 9 9 4 , 5 6 4 7 , 3 8 3 0 - 3 8 3 6 .}$

66 D. Volodkin and R. von Klitzing, Curr. Opin. Colloid Interface Sci., 2014, 19, 25-31.

67 N. G. Hoogeveen, M. A. Cohen Stuart, G. J. Fleer and M. R. Boehmer, Langmuir, 1996, 7463, 3675-3681.

68 V. Kabanov and A. Kabanov, Adv. Drug Delivery Rev., 1998, 30, 49-60.

69 J. B. Schlenoff, A. H. Rmaile and C. B. Bucur, J. Am. Chem. Soc., 2008, 130, 13589-13597.

70 B. Qiao, J. J. Cerda and C. Holm, Macromolecules, 2010, 43, 7828-7838.

71 B. N. Balzer, S. Micciulla, S. Dodoo, M. Zerball, M. Gallei, M. Rehahn, R. V Klitzing and T. Hugel, ACS Appl. Mater. Interfaces, 2013, 5, 6300-6306. 